\title{
Niveles enzimáticos de lactato deshidrogenasa en saliva según la condición periodontal
}

\author{
Enzyme levels of lactate dehydrogenase in saliva according to the condition periodontal
}

\author{
Sharon Miranda-Quispe ${ }^{1, a}$, María Pareja-Vásquez ${ }^{1, a, b}$, Manuel Antonio Mattos-Velaa, ${ }^{1, a, c}$.
}

\section{RESUMEN}

Objetivo: Comparar los niveles enzimáticos de lactato deshidrogenasa (LDH) en la saliva según la condición periodontal. Material y métodos: Estudio descriptivo, transversal. Se incluyeron 40 pacientes entre 23 y 73 años de edad, de una clínica especializada en odontología. Hubo 10 pacientes en cada grupo: uno control (periodontalmente sanos) y tres de estudio. Las muestras de saliva no estimulada $(2 \mathrm{ml})$ fueron recogidas en frascos para muestra de esputo, directamente de la boca; se trasladaron a tubos Eppendorf de $2 \mathrm{ml}$ y se centrifugaron a $300 \mathrm{rpm}$ por 10 minutos. Para evaluar la actividad enzimática, fueron colocados en el analizador automático "Vitalab Selectra II" (vital cientific, Holanda) con el reactivo LDH-P UV AA líquido, para muestras de suero o plasma. El dinucleótido de nicotinamida y adenina (NADH) consumido, fue directamente proporcional a la actividad de LDH en la muestra de saliva. Los datos se analizaron con las pruebas de Kruskal-Wallis y post hoc de Dunn-Bonferroni. Resultados: Se encontró diferencias significativas en los niveles de LDH entre los pacientes con periodontitis crónica generalizada frente a los periodontalmente sanos y en fase de mantenimiento ( $<<0,01)$. También hubo diferencia significativa $(\mathrm{p}=0,006)$ entre los valores de LDH de los pacientes con periodontitis crónica localizada y los periodontalmente sanos. Conclusiones: Los niveles enzimáticos de LDH están disminuidos en los pacientes en fase de mantenimiento periodontal respecto a los pacientes con periodontitis crónica generalizada, así mismo estos valores son muy cercanos a los pacientes periodontalmente sanos y con periodontitis crónica localizada.

PALABRAS CLAVE: Periodontitis, enzimas, L-lactato deshidrogenasa, saliva, diagnóstico.

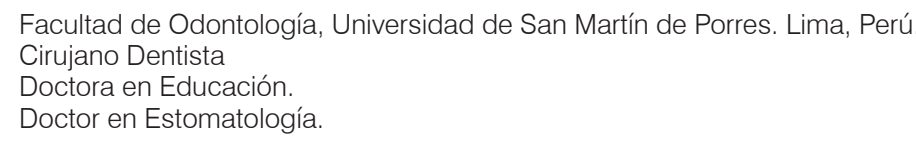




\section{SUMMARY}

Objective: To compare those levels enzyme of lactate dehydrogenase (LDH) in the saliva according to the condition periodontal. Material and methods: Descriptive, cross-sectional study. It was included 40 patients between 23 and 73 years, of a clinic specializing in dentistry. There were 10 patients in each group: one control (periodontally healthy) and three study. Not stimulated saliva samples $(2 \mathrm{ml})$ were collected in vials for sample of sputum, directly from the mouth; they moved to $2 \mathrm{ml}$ Eppendorf tubes and centrifuged at $300 \mathrm{rpm}$ for 10 minutes. To assess enzymatic activity, they were placed in the automatic Analyzer "Vitalab Selectra II" (life science, the Netherlands) with reagents LDH-P UV AA liquid for serum or plasma samples. The dinucleotide of NICOTINAMIDE and adenine (NADH) consumed, was directly proportional to the activity of LDH in the saliva samples. The data were analyzed with post hoc Bonferroni-Dunn and Kruskal-Wallis tests. Results: It was found significant differences in the levels of LDH among patients with generalized chronic periodontitis versus periodontally healthy and in phase of maintenance $(p<0.01)$. Also there was difference significant $(p=$ 0.006) between the values of LDH of them patients with localized chronic periodontitis and the periodontally healthy. Conclusions: Enzyme LDH levels are decreased in patients during periodontal maintenance in respect to patients that have generalized chronic periodontitis, also these values are very close to periodontally healthy patients and localized chronic periodontitis.

\section{KEY WORDS: Periodontitis, enzymes, L-lactate dehydrogenase, saliva, diagnosis.}

\section{INTRODUCCIÓN}

En la actualidad las periodontopatías constituyen un problema de salud pública, tienen alta prevalencia, impacto social y generan un costo importante por su tratamiento $(1,2)$.

La periodontitis, es una enfermedad inflamatoria de origen infeccioso, resultante de las complejas interacciones de los patógenos periodontales de la biopelícula dental localizados en el surco gingival y la presencia de un hospedero susceptible (3). Se caracteriza por la presencia de inflamación pérdida de inserción clínica y reabsorción del hueso alveolar (3), es multifactorial y una de las principales causas de pérdida de dientes en adultos mayores de 35 años, y se ha estimado que hasta el $90 \%$ de la población adulta mundial puede tener por lo menos una forma leve de enfermedad periodontal $(4,5)$. Por lo tanto, para ejecutar un tratamiento periodontal exitoso es necesario determinar de forma adecuada el diagnóstico y pronóstico periodontal.

Los métodos de diagnóstico tradicionales nos permiten determinar los cambios en el nivel de inserción clínica y pérdida ósea; sin embargo se han desarrollado métodos complementarios de diagnóstico, a través de pruebas bioquímicas, para detectar la actividad de la enfermedad periodontal $(3,6)$.
Los fluidos corporales como la saliva y el fluido crevicular gingival están siendo utilizados para evaluar enfermedades y realizar diagnósticos clínicos $(7,8)$. Existen estudios en los que se reporta que la saliva y el fluido crevicular gingival son de utilidad para el diagnóstico de la enfermedad periodontal, debido a que se han identificado diversos marcadores que se podrían utilizar en la clínica, a fin de evaluar la progresión de esta enfermedad (3,6,9-11).

Se ha encontrado que algunas enzimas intracelulares procedentes de las células periodontales dañadas, incrementan su liberación durante el proceso de enfermedad periodontal, pudiendo localizarse en la saliva y el líquido crevicular. Las enzimas particularmente relevantes en este grupo son: lactato deshidrogenasa (LDH) (9), aspartato y alanino aminotransferasa, gama-glutamil-transferasa, creatin-kinasa, alcalin-fosfatasa y ácido-fosfatasa. Algunos estudios reportan la utilidad de estas enzimas para medir la actividad de la enfermedad periodontal o para valorar su tratamiento (11-13). Estas enzimas pueden ser detectadas en muestras de saliva, con técnicas como la espectrofotometría y la electroforesis(11).

La LDH y la aspartato amino transferasa pueden ayudar a monitorear la progresión de la enfermedad periodontal. Estas enzimas intracitoplasmáticas aumentan con la destrucción celular o tisular (6), pueden considerarse como biomarcadores $(8,14)$. 
La LDH es una enzima intracelular, que se encuentra en el citoplasma de la mayoría de células del cuerpo humano. La elevación de los niveles de LDH en el plasma sanguíneo evidencia la salida de esta enzima al espacio extracelular, lo que se produce cuando hay muerte celular. Por lo tanto, los niveles elevados de LDH, indican daño tisular o necrosis celular (15).

La actividad de la LDH salival, se relaciona con el daño en los tejidos de la cavidad bucal, como la inflamación gingival y la destrucción de los tejidos de soporte en la enfermedad periodontal (8); variación que es detectable tanto en pacientes fumadores como no fumadores (16). A la vez la LDH salival es considerada un biomarcador potencial para detectar y diagnosticar carcinoma oral de células escamosas $(17,18)$. La LDH se descompone en cinco isoenzimas: LDH1, LDH-2, LDH-3, LDH-4 y LDH-5 las que pueden identificarse a través de electroforesis. Estas isoenzimas pueden encontrarse también en la saliva (19).

Diversos estudios se han sido realizados con el objetivo de determinar la actividad de la LDH en la saliva de pacientes con enfermedad periodontal y en pacientes sanos, encontrándose que el aumento de los niveles de LDH está asociado con la presencia de enfermedad periodontal, especialmente si hay cálculo dental y bolsas de más de $5 \mathrm{~mm}(9)$.

También se reportan estudios en los cuales se ha evaluado la actividad de enzimas salivales como el aspartato y alanina aminotransferasa, creatina cinasa, fosfatasa ácida y alcalina, gama glutamil transferasa y LDH en la saliva de pacientes con enfermedad periodontal, antes y después del tratamiento periodontal, así como en la saliva de pacientes sanos; observaron un aumento estadísticamente significativo en la actividad de las enzimas examinadas, en la saliva de los pacientes con enfermedad periodontal con respecto a los pacientes sanos. Una reducción significativa en los niveles de estas enzimas fue visto después de la terapia periodontal convencional (11).

Se han realizado diversos estudios con el fin de demostrar la utilidad del fluido crevicular gingival y la saliva para la detección de la enfermedad periodontal $(9,19-21)$. Uno de ellos reporta que la medición de los niveles de LDH salival, si bien es un método menos sensible y especifico que el Índice de Necesidad de Tratamiento Periodontal de la Comunidad (CPITN), para identificar la enfermedad periodontal; es menos invasivo y es una alternativa para la detección masiva de esta enfermedad (10).

El fluido crevicular gingival muestra evidencias sobre su utilidad (21), sin embargo, la toma de muestra puede ser más compleja; por el contrario, para obtener muestras de saliva, la técnica es mucho más simple, es un proceso no invasivo y de menor costo (10). Es importante el conocimiento de las variaciones de los niveles enzimáticos según el estado periodontal del paciente, así como el establecimiento de patrones estandarizados de acuerdo a los valores encontrados. Esta información permitirá utilizar los biomarcadores como un método auxiliar de diagnóstico y seguimiento de la enfermedad periodontal.

El objetivo de este estudio fue comparar los niveles enzimáticos de LDH en la saliva según la condición periodontal.

\section{MATERIAL Y MÉTODOS}

Fue un estudio descriptivo, transversal. Fueron incluidos 40 pacientes de ambos sexos, de 23 a 73 años, con un mínimo de 10 dientes naturales en boca, atendidos en la Maestría de Periodoncia, de la Sección de Posgrado de la Universidad de San Martín de Porres (USMP), entre los meses de mayo y julio del 2013. Todos los pacientes que participaron firmaron un consentimiento informado de acuerdo al Instituto de investigación de la Facultad de Odontología de la USMP. Previo a la toma de las muestras se realizó historia clínica y un examen periodontal a los pacientes participantes empleando la sonda de la OMS, marca Hu Friedy (USA). Una sola persona, entrenada en los criterios a evaluar, realizó un periodontograma midiendo el nivel de inserción clínica, la profundidad, y el sangrado al sondeo. Se tomó como parámetro para definir el diagnostico de periodontitis el criterio de la Federación Europea de Periodoncia (EFP, 2005) (15): $\geq$ a 2 sitios independientes con pérdida de inserción de $\geq$ a $3 \mathrm{~mm}$ proximal y formación de bolsa periodontal. Los pacientes en fase de mantenimiento fueron aquellos dados de alta de su tratamiento periodontal, debían estar en su segundo control de 6 meses a más, con un índice de O'Leary de $20 \%$ o menos. Se consideró como periodontitis crónica localizada si el paciente tenía $30 \%$ o menos de sitios afectados y generalizada si el porcentaje era mayor de $30 \%$. 
Fueron excluidos del estudio pacientes bajo tratamiento médico o periodontal, con enfermedades sistémicas, fumadores, con terapia antibiótica, gestantes y con tratamiento de ortodoncia fija. Los pacientes fueron distribuidos en cuatro grupos de 10 pacientes cada uno: un grupo control (periodontalmente sanos), y tres grupos de estudio conformados por pacientes diagnosticados con periodontitis crónica localizada, con periodontitis crónica generalizada y en terapia periodontal de mantenimiento.

\section{Procedimientos y técnicas}

Previo enjuagatorio con $15 \mathrm{ml}$ de agua, para eliminar células exfoliadas, se recogió directamente las muestras de saliva sin estimulación, luego de ser acumuladas en el piso de boca. El horario de toma de muestra se efectuó entre 9:00 y 13:00 horas. La cantidad recolectada fue aproximadamente $2 \mathrm{ml}$ por paciente en frascos de muestra de esputo codificados, que fueron almacenados a baja temperatura $\left(4^{\circ} \mathrm{C}\right)$ en un cooler (Mega, Perú), acondicionados con cuatro bolsas de gel refrigerante "Rubbermaid" (USA), la temperatura fue controlada, medida y monitorizada con un termómetro de alcohol "Giardino" (Perú).

El mismo día que se recolectaron las muestras, fueron llevadas al laboratorio de Bioquímica y Nutrición de la Facultad de Medicina de la USMP.

Las muestras almacenadas a $4{ }^{\circ} \mathrm{C}$ se trasladaron a tubos Eppendorf de $2 \mathrm{ml}$ y se colocaron en la centrífuga Heraeus Labofuge 400 (Alemania) a $300 \mathrm{rpm}$ por 10 minutos para separar los contaminantes sólidos.

Luego 500 ul del sobrenadante de cada tubo se trasladaron mediante micropipetas "Transferpette" (Alemania) a 8 colectores de polietileno, utilizado en lugar de tubos de vidrio para facilitar el análisis de la muestra. Estas fueron posteriormente colocadas en el analizador automático "Vitalab Selectra II" (vital cientific, Holanda) con el reactivo LDH-P UV AA líquido, del laboratorio Wiener lab. (Argentina) para muestras del suero o plasma, a fin de evaluar la actividad enzimática.

En presencia de LDH el piruvato fue reducido a lactato con la oxidación simultánea del dinucleótido de nicotinamida y adenina, en su forma reducida (NADH). El NADH consumido fue directamente proporcional a la actividad de $\mathrm{LDH}$ en la muestra de saliva. Las concentraciones del ensayo estuvieron calibradas de acuerdo a la Sociedad Francesa de Biología Clínica.

Los datos fueron procesados y analizados en el programa estadístico SPSS v21. Para comparar la semejanza de los grupos de estudio se aplicaron las pruebas de ANOVA de un factor (para las variables edad y número de piezas dentarias presentes) y exacta de Fisher (para el sexo). Se realizó el análisis descriptivo del nivel enzimático del lactato deshidrogenasa en cada grupo de estudio y control a través de la mediana como medida de centro y el rango intercuartílico como medida de dispersión junto con los valores mínimo y máximo. Previo al análisis inferencial se determinó la presencia de distribución normal en los datos por medio de la prueba de Shapiro-Wilk.

Luego, para realizar la comparación de la variable de estudio entre todos los grupos se aplicó la prueba de Kruskal-Wallis seguido de pruebas post hoc de Dunn-Bonferroni para las comparaciones por pares. Todas las pruebas se trabajaron a un nivel de significancia de $5 \%$.

\section{RESULTADOS}

La muestra final estuvo integrada por 40 pacientes, 10 en cada grupo. La descripción de los pacientes de cada uno de los grupos respecto a la edad, sexo, número de piezas dentarias presentes y nivel enzimático se presenta en la tabla 1. Las características de los grupos de estudio fueron semejantes $(p>0,05)$, siendo la edad media mayor a los 50 años, con predominio del sexo femenino y aproximadamente 20 piezas dentales presentes en boca. El grupo control estuvo conformado por pacientes más jóvenes con una edad media 30,9 años (tabla 2).

Cuando se evaluó el nivel enzimático del lactato deshidrogenasa en cada grupo de estudio y control se encontró que el mayor valor mediano (1469,5 U/1) y de dispersión: rango intercuartílico $(\mathrm{RI})=1657$ se ubicó en el grupo con periodontitis crónica generalizada, lo cual fue estadísticamente diferente $(\mathrm{p}<0,01)$ a los valores obtenidos por los grupos periodontalmente sanos (mediana $259 \mathrm{U} / 1$ y $\mathrm{RI}=107 \mathrm{U} / 1$ ) y en fase de mantenimiento periodontal (mediana $299 \mathrm{U} / \mathrm{I}$ y RI= $125 \mathrm{U} / \mathrm{I})$, pero semejante $(\mathrm{p}=0,976)$ al de la periodontitis crónica localizada (mediana $866 \mathrm{U} / \mathrm{I}$ y $\mathrm{RI}=675$ 
Tabla 1. Características de los pacientes de cada grupo

\begin{tabular}{|c|c|c|c|c|c|}
\hline Grupo & Paciente & Edad & Sexo* & $\begin{array}{c}\text { Piezas } \\
\text { presentes }\end{array}$ & $\begin{array}{c}\text { Nivel } \\
\text { enzimático (U/l) }\end{array}$ \\
\hline Periodontalmente & 1 & 65 & M & & 259 \\
\hline \multirow{9}{*}{ sanos } & 2 & 31 & M & & 163 \\
\hline & 3 & 29 & $\mathrm{~F}$ & & 289 \\
\hline & 4 & 28 & M & & 177 \\
\hline & 5 & 27 & M & & 291 \\
\hline & 6 & 25 & M & & 276 \\
\hline & 7 & 25 & M & & 145 \\
\hline & 8 & 30 & $\mathrm{~F}$ & & 259 \\
\hline & 9 & 24 & $\mathrm{~F}$ & & 278 \\
\hline & 10 & 25 & $\mathrm{~F}$ & & 195 \\
\hline Periodontitis & 1 & 60 & M & 19 & 1154 \\
\hline crónica & 2 & 68 & $\mathrm{~F}$ & 18 & 588 \\
\hline \multirow[t]{8}{*}{ localizada } & 3 & 48 & $\mathrm{~F}$ & 24 & 1203 \\
\hline & 4 & 72 & $\mathrm{~F}$ & 15 & 818 \\
\hline & 5 & 23 & M & 24 & 205 \\
\hline & 6 & 35 & $\mathrm{~F}$ & 27 & 1052 \\
\hline & 7 & 74 & $\mathrm{~F}$ & 22 & 521 \\
\hline & 8 & 64 & $\mathrm{~F}$ & 24 & 914 \\
\hline & 9 & 29 & $\mathrm{~F}$ & 25 & 401 \\
\hline & 10 & 50 & $\mathrm{~F}$ & 19 & 1761 \\
\hline Periodontitis & 1 & 59 & $\mathrm{~F}$ & 23 & 1660 \\
\hline crónica & 2 & 72 & $\mathrm{~F}$ & 15 & 715 \\
\hline \multirow[t]{8}{*}{ generalizada } & 3 & 63 & $\mathrm{~F}$ & 21 & 3579 \\
\hline & 4 & 41 & M & 30 & 1279 \\
\hline & 5 & 77 & $\mathrm{~F}$ & 11 & 1845 \\
\hline & 6 & 63 & $\mathrm{~F}$ & 16 & 619 \\
\hline & 7 & 55 & M & 29 & 557 \\
\hline & 8 & 67 & $\mathrm{~F}$ & 28 & 3246 \\
\hline & 9 & 61 & M & 26 & 1204 \\
\hline & 10 & 41 & $\mathrm{~F}$ & 25 & 2049 \\
\hline Fase de & 1 & 61 & $\mathrm{~F}$ & 24 & 658 \\
\hline mantenimiento & 2 & 53 & $\mathrm{~F}$ & 16 & 274 \\
\hline \multirow[t]{8}{*}{ periodontal } & 3 & 43 & $\mathrm{~F}$ & 21 & 310 \\
\hline & 4 & 71 & $\mathrm{~F}$ & 15 & 367 \\
\hline & 5 & 66 & $\mathrm{~F}$ & 19 & 127 \\
\hline & 6 & 73 & $\mathrm{~F}$ & 10 & 335 \\
\hline & 7 & 43 & M & 24 & 255 \\
\hline & 8 & 53 & $\mathrm{~F}$ & 21 & 288 \\
\hline & 9 & 61 & $\mathrm{~F}$ & 27 & 269 \\
\hline & 10 & 68 & $\mathrm{~F}$ & 22 & 459 \\
\hline Media $\_D E$ & & $50,6 \pm 17,7$ & & $21,3 \pm 5,2$ & \\
\hline Mediana $\_\mathrm{RI}^{\dagger}$ & & & & & $430 \pm 858,3$ \\
\hline
\end{tabular}

*Sexo: $\mathrm{F}=$ Femenino, $\mathrm{M}=$ Masculino $\dagger \mathrm{RI}=$ Rango intercuartílico

$\uparrow \mathrm{RI}=$ Rango intercuartílico 
Tabla 2. Características de los grupos control y estudio de acuerdo a edad, sexo y número de piezas dentarias presentes.

\begin{tabular}{|c|c|c|c|c|c|}
\hline Características & $\begin{array}{c}\text { Periodontalmente } \\
\text { sanos }\end{array}$ & $\begin{array}{c}\text { Periodontitis } \\
\text { crónica } \\
\text { localizada }\end{array}$ & $\begin{array}{c}\text { Periodontitis } \\
\text { crónica } \\
\text { generalizada }\end{array}$ & $\begin{array}{c}\text { Fase de } \\
\text { mantenimiento } \\
\text { periodontal }\end{array}$ & Valor $\mathbf{p}^{*}$ \\
\hline $\mathrm{n}$ & 10 & 10 & 10 & 10 & \\
\hline Edad (media_DE) & $30,9 \pm 12,2$ & $52,3+18,3$ & $59,9+11,8$ & $59,2+10,9$ & 0,421 \\
\hline Sexo & & & & & 0,847 \\
\hline Femenino & 4 & 8 & 7 & 9 & \\
\hline Masculino & 6 & 2 & 3 & 1 & \\
\hline Piezas presentes (media $\_$DE) & & $21,7+3,8$ & $22,4+6,5$ & $19,9+5,0$ & 0,551 \\
\hline
\end{tabular}

Tabla 3. Nivel enzimático de lactato deshidrogenasa en los grupos control y de estudio.

\begin{tabular}{|c|c|c|c|c|c|c|}
\hline \multirow[b]{2}{*}{ Grupo } & \multirow[b]{2}{*}{$\mathbf{n}$} & \multicolumn{3}{|c|}{ Nivel enzimático (U/l) } & \multirow[b]{2}{*}{$\mathbf{R I}^{*}$} & \multirow[b]{2}{*}{ Valor $\mathrm{p}$} \\
\hline & & Mínimo & Máximo & Mediana $^{\dagger}$ & & \\
\hline Periodontalmente sanos & 10 & 145 & 291 & $259^{\mathrm{a}}$ & 107 & $<0,001$ \\
\hline Periodontitis crónica localizada & 10 & 205 & 1761 & $866^{\mathrm{b}, \mathrm{c}}$ & 675 & \\
\hline Periodontitis crónica generalizada & 10 & 557 & 3579 & $1469,5^{\mathrm{c}}$ & 1657 & \\
\hline Fase de mantenimiento periodontal & 10 & 127 & 658 & $299^{\mathrm{a}, \mathrm{b}}$ & 125 & \\
\hline
\end{tabular}

* RI= Rango intercuartílico

$\dagger$ Letras indican subgrupos homogéneos, dentro de los cuales no hay diferencia estadísticamente significativa

$\mathrm{U} / \mathrm{I})$. Se encontró diferencia significativa $(\mathrm{p}=0,006)$ entre los grupos periodontalmente sanos y con periodontitis crónica localizada. No hubo diferencia entre los valores del grupo de fase de mantenimiento periodontal con los periodontalmente sanos y periodontitis crónica localizada (valores $\mathrm{p}=1$ y 0,175 respectivamente) (tabla 3, gráfico 1).

\section{DISCUSIÓN}

Los tres grupos de estudio fueron semejantes en cuanto a la edad, sexo y número de piezas dentarias presentes, sin embargo, el grupo control estuvo conformado por pacientes más jóvenes (edad media 30,9 años), puesto que no se pudo encontrar personas mayores periodontalmente sanas, lo cual representa una limitación del estudio. A pesar de ello, permite tener un primer acercamiento a los niveles enzimáticos de LDH en un grupo de personas sanas.

Este estudio tuvo como objetivo determinar los niveles enzimáticos de LDH en saliva de pacientes según la condición periodontal. Sus principales resultados muestran que existen diferencias entre los niveles de LDH en pacientes con periodontitis crónica generalizada comparada con pacientes sanos y en fase de mantenimiento y entre pacientes con periodontitis crónica localizada y pacientes sanos. 


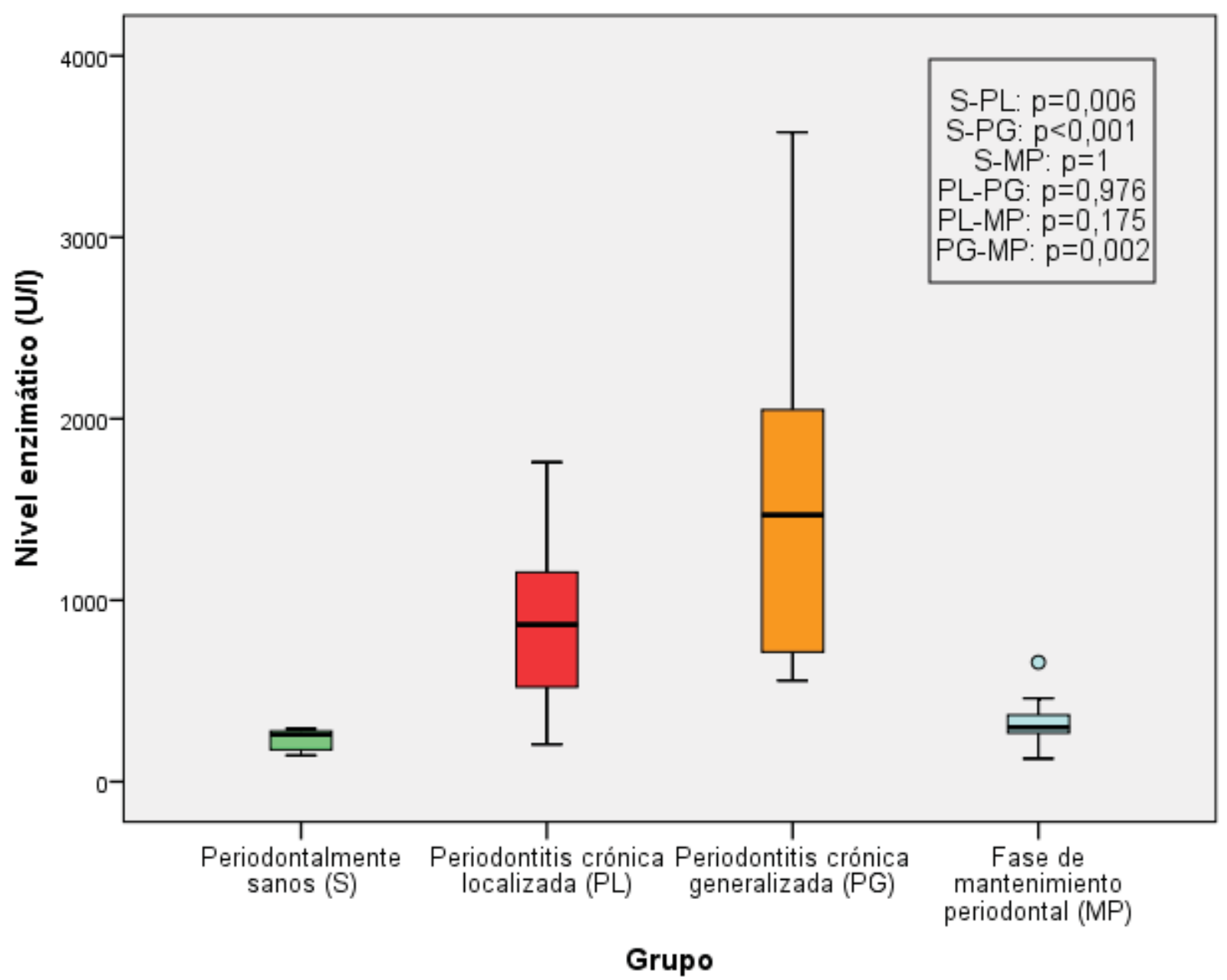

Gráfico 1. Nivel enzimático de lactato deshidrogenasa entre los diversos grupos.

Se confirma que las enzimas salivales pueden ser consideradas como marcadores bioquímicos para determinar las condiciones periodontales, lo que coincide con la mayoría de estudios que evalúan este aspecto $(3,6,9,11-13,15)$.

En el presente estudio se evaluaron pacientes con diferentes condiciones periodontales, periodontitis crónica localizada, periodontitis crónica generalizada, en fase de mantenimiento y sanos. Se observó que los pacientes en fase de mantenimiento periodontal presentaban niveles bajos de LDH muy cercanos a los valores de pacientes periodontalmente sanos y que los pacientes con periodontitis crónica localizada y generalizada por el contrario presentaban valores más elevados de LDH.
Nomura et al., determinaron la utilidad de los marcadores bioquímicos salivales para detectar la enfermedad periodontal y contrastaron los resultados de las pruebas de enzimas salivales frente a los de profundidad de sondaje en 187 sujetos (19). Ellos encontraron que el nivel de LDH salival tuvo mayor sensibilidad y especificidad (sensibilidad 0.66, especificidad 0.67), que los niveles salivales de aspartato aminotransferasa (AST) y nitrógeno ureico en sangre (BUN). Concluyeron que la LDH salival puede ser un parámetro viable y útil para detectar de la enfermedad periodontal (19). Se encuentran coincidencias con este estudio, considerando que la medición de LDH en los pacientes evaluados, permitió diferenciar claramente los valores de los grupos de periodontitis crónica localizada y generalizada con el grupo de pacientes sanos. 
Según Todorovic et al., la actividad de las enzimas de la saliva de pacientes con enfermedad periodontal fue significativamente mayor en relación con el grupo control (11). En este estudio se evidenció también que los pacientes diagnosticados con periodontitis crónica presentaron niveles enzimáticos de LDH significativamente aumentados.

Puede citarse el estudio realizado por Ali et al., en 200 participantes, divididos en cuatro grupos según su estado periodontal y hábito de fumar, el cual tuvo como objetivo establecer si las enzimas lactato deshidrogenasa (LDH) y la $\beta$-glucuronidasa obtenidas de saliva no estimulada eran eficaces como biomarcadores para la periodontitis se encontró un aumento significativo en la actividad de las enzimas en los grupos de periodontitis, en comparación con los grupos que no presentaban periodontitis ( $p<0,001$ ), y concluyeron que aun cuando el fumar influye en la actividad de las enzimas LDH y la $\beta$-glucuronidasa, estas son biomarcadores salivales confiables de periodontitis (22), estos resultados coinciden con los del presente estudio, específicamente con respecto a la eficacia de la enzima LDH.

Así mismo, Mohanty et al., compararon los niveles de LDH en saliva, como biomarcador, en 80 participantes, divididos en diferentes grupos de fumadores, para el diagnóstico temprano de periodontitis, observaron una correlación significativa entre los valores de LDH salival y la condición periodontal evaluada mediante el Índice de Necesidad de Tratamiento Periodontal de la Comunidad (CPITN), coincidiendo con los resultados obtenidos en esta investigación (23).

Taniguchi-Tabata et al., en el 2019, también utilizaron los niveles de LDH obtenida de saliva no estimulada para evaluar la condición gingival en 1915 adultos jóvenes japoneses y contrastarlos con factores asociados. Para determinar la presencia de gingivitis consideraron un nivel de LDH de 8 8 ; además utilizaron de manera eficaz los niveles de esta enzima para su estudio, lo que corrobora que es una herramienta eficaz para esta medición (24).

Las limitaciones principales de este estudio fueron el tamaño de la muestra, sería conveniente ampliar el número de sujetos evaluados; que fue un estudio transversal, sería importante realizar un estudio longitudinal, comparando los valores de antes y después del tratamiento periodontal, y continuar la medición en la fase de mantenimiento.

Se concluye que los niveles enzimáticos de LDH en saliva de pacientes en fase de mantenimiento periodontal están disminuidos respecto a los valores de los pacientes con periodontitis crónica generalizada, pero son semejantes en comparación a los pacientes periodontalmente sanos y con periodontitis crónica localizada. Los niveles enzimáticos de LDH son menores en los pacientes periodontalmente sanos respecto a los pacientes con periodontitis crónica localizada y generalizada. No se hallaron diferencias en los niveles enzimáticos de LDH en saliva de pacientes con periodontitis crónica localizada y pacientes con periodontitis crónica generalizada. Los resultados de este estudio podrían aplicarse para evaluar la condición periodontal de los pacientes diagnosticados con periodontitis que hayan sido sometidos a terapia periodontal. Un análisis de una muestra de saliva en cada visita de control durante la fase de mantenimiento podría proveer datos importantes para el periodoncista o el odontólogo general. Tales pruebas bioquímicas tendrían que considerarse como un análisis complementario a la evaluación clínica periodontal.

\section{Correspondencia:}

María Pareja-Vásquez\}

Correo electrónico: mariapareja-@hotmail.com

\section{REFERENCIAS BIBLIOGRÁFICAS}

1. Batchelor P. Is periodontal disease a public health problem? Br Dent J. 2014;217(8):405-9. doi: 10.1038/ sj.bdj.2014.912

2. Sexton WM, Lin Y, Kryscio RJ, Dawson DR, Ebersole JL, Miller CS. Salivary biomarkers of periodontal disease in response to treatment. J Clin Periodontol. 2011;38(5):434-441. doi: 10.1111/j.1600051X.2011.01706.x

3. Scapoli L, Girardi A, Palmieri A, et al. IL6 and IL10 are genetic susceptibility factors of periodontal disease. Dent Res J. 2012; 9(Suppl. 2):S197-S201. doi: 10.4103/1735-3327.109754

4. You M, Mo S, Leung WK, Watt RM. Comparative analysis of oral treponemes associated with periodontal health and disease. BMC Infect Dis. 2013;13:174.

5. Castro CE, Koss MA, López ME. Intracytoplasmic enzymes in gingival crevicular fluid of patients with aggressive periodontitis. J Periodontal Res 2011;46(5):522-7. 
6. Alfaqeeh SA, Anil S. Lactate dehydrogenase activity in gingival crevicular fluid as a marker in orthodontic tooth movement. Open Dent J. 2011;5:105-9.

7. Javaid MA, Ahmed AS, Durand R, Tran SD. Saliva as a diagnostic tool for oral and systemic diseases. J Oral Biol Craniofac Res. 2016;6(1):66-75.

8. De La Peña VA, Dios PD, Sierra RT. Relationship between lactate dehydrogenase activity in saliva and oral health status. Arch Oral Biol. 2007;52(10):911-5.

9. Dabra S, China K, Kaushik A. Salivary enzymes as diagnostic markers for detection of gingival/periodontal disease and their correlation with the severity of the disease. J Indian Soc Periodontol. 2012;16(3):358-364.

10. Nomura Y, Okada A, Kakuta E, Gunji T, Kajiura S, Hanada N. A new screening method for periodontitis: an alternative to the community periodontal index. BMC Oral Health. 2016; 16(1):64.

11. Todorovic T, Dozic I, Vicente-Barrero M, et al. Salivary enzymes and periodontal disease. Med Oral Patol Oral Cir Bucal. 2006;11(2):E115-9.

12. Khiste SV, Ranganath V, Nichani AS, Rajani V. Critical analysis of biomarkers in the current periodontal practice. J Indian Soc Periodontol. 2011;15(2):104-10.

13. Sundar NM, Krishnan V, Krishnaraj S, Hemalatha VT, Alam MN. Comparison of the salivary and the serum nitric oxide levels in chronic and aggressive periodontitis: A biochemical study. J Clin Diagn Res. 2013;7(6):1223-7.

14. Tonetti MS, Claffey N. Advances in the progression of periodontitis and proposal of definitions of a periodontitis case and disease progression for use in risk factor research. Group C consensus report of the $5 \mathrm{Eu}-$ ropean Workshop in Periodontology. J Clin Periodontol. 2005; 32 (Suppl. 6):205-208.

15. Nagler RM, Lischinsky S, Diamond E, Klein I, Reznick AZ. New insights into salivary lactate dehydrogenase of human subjects. J Lab Clin Med 2001;137:363-9.

16. Rao K, Babu SG, Shetty UA, Castelino RL, Shetty SR. Serum and salivary lactate dehydrogenase levels as biomarkers of tissue damage among cigarette smokers. A biochemical study. Stomatologija. 2017;19(3): 91-96.

17. Lokesh K, Kannabiran J, Rao MD. Salivary Lactate Dehydrogenase (LDH)- A Novel Technique in Oral Cancer Detection and Diagnosis. J Clin Diagn Res. 2016;10(2):ZC34-7.

18. Pathiyil V, D'Cruz AM. Salivary lactate dehydrogenase as a prognostic marker in oral squamous cell carcinoma patients following surgical therapy. J Exp Ther Oncol. 2017; 11(2): 133-137.

19. Nomura Y, Tamaki Y, Tanaka T, et al. Screening of periodontitis with salivary enzyme tests. J Oral Sci. 2006;48(4):177-83.

20. Luke R, Khan SN, Iqbal PS, Soman RR, Chakkarayan J, Krishnan V. Estimation of specific salivary enzy- matic biomarkers in individuals with gingivitis and chronic periodontitis: A clinical and biochemical study. J Int Oral Health. 2015;7(9):54-7.

21. González J, Rivera S. Efecto del tratamiento periodontal no quirúrgico sobre los niveles de Piridinolina (ICTP) en el fluido gingival crevicular (FGC). ODOVTOS-Int J Dental Sc. 2017; 19(1): 77-85. DOI: $10.15517 /$ ijds.v0i0.27465

22. Ali SA, Telgi RL, Tirth A, Tantry IQ, Aleem A. Lactate Dehydrogenase and $\beta$-glucuronidase as salivary biochemical markers of periodontitis among smokers and non-smokers. Sultan Qaboos Univ Med J. 2018;18(3):e318-e323. doi:10.18295/ squmj.2018.18.03.009

23. Mohanty P, Gujjari SK, Nakum CG. Salivary free hemoglobin and lactate dehydrogenase as biomarkers for periodontal disease in smokers. Quintessence Int. 2019;50(6):428-434. doi: 10.3290/j.qi.a42478.

24. Taniguchi-Tabata A, Ekuni D, Azuma T, et al. The level of salivary lactate dehydrogenase as an indicator of the association between gingivitis and related factors in Japanese university students. J Oral Sci. 2019;61(1):133-139. doi:10.2334/josnusd.18-0038.

Recibido: 16-09-2018

Aceptado: 23-01-2019 